\title{
Erratum to: The Effects of DSM5 Autism Diagnostic Criteria on Number of Individuals Diagnosed with Autism Spectrum Disorders: A Systematic Review
}

Peter Sturmey • Samantha Dalfen

Published online: 21 August 2014

(C) Springer Science+Business Media New York 2014

Erratum to: Review Journal of Autism and Developmental Disorders

DOI 10.1007/s40489-014-0016-7

Please note that the surname of the second author of this article was misspelled in the published article.

The correct surname of the second author is Dalfen (NOT "Dalfern").

The online version of the original article can be found at http://dx.doi.org/ 10.1007/s40489-014-0016-7.

P. Sturmey $\cdot$ S. Dalfen

Queens College, City University of New York, New York, NY, USA

P. Sturmey $(\bowtie) \cdot$ S. Dalfen

Queens College, City University of New York, New York, NY, USA

e-mail: Peter.Sturmey@qc.cuny.edu 